\title{
Fiber optics sensor for sub-nanometric displacement and wide bandwidth systems
}

\author{
L. Perret, L Chassagne*, S. Topçu, P. Ruaux, B. Cagneau, Y. Alayli \\ Laboratoire d'Ingénierie des Systèmes de Versailles, Université de Versailles Saint-Quentin, 45 \\ avenue des Etats-Unis, 78035 Versailles, France \\ Corresponding author: luc.chassagne@uvsq.fr +3313925 4478 \\ Exact reference : \\ L. Perret, L. Chassagne, S. Topcu, P. Ruaux, B. Cagneau, Y. Alayli, Fiber optics sensor for sub-nanometric \\ displacement and wide bandwidth systems, Sensors and Actuators A, 165, 189-193 (2011).
}

\begin{abstract}
In this paper, we report fiber optics sensor with sub-nanometric resolution and wide bandwidth. It relies on an increase of the reception fibers number and on low-noise electronics. Moreover, a reference channel has been implemented using a semi-reflective plate to eliminate the source fluctuations and the fiber sensor was isolated to limit external influence of temperature and pressure. Thus we achieve both a sub-nanometric resolution on a $400 \mathrm{~ms}$ integration time and a long-term drift as low as $40 \mathrm{~nm} \cdot \mathrm{h}^{-1}$. The setup has been also adapted to high speed applications by increasing the bandwidth up to $38 \mathrm{kHz}$. It can display a $28 \mathrm{~nm}$ peak-to-peak limit of resolution on an aluminized piezoactuator. It has been successfully used to test the resonance frequency of a vibrating plate actuated by two highfrequency prototypes of piezoactuators. These improvements lead to low cost fibers optic sensors interesting for non-contact displacement measurements with high sensitivity.
\end{abstract}

Keywords: Fiber optics, high resolution sensor, distance measurement, vibration measurement, high bandwidth sensor, low-noise electronics.

\section{Introduction}

Many non-contact measurement systems have been explored to reach the sub-nanometric resolution, in order to satisfy the need in nanopositioning and nanomanipulation fields such as Atomic Force Microscopy (AFM) and Scanning Near-Field Optical Microscopy (SNOM), electronic lithography, atomic and molecular physics, or to characterize low amplitudes vibrations and to achieve high-speed servo-loops. The most efficient systems are based on interferometry but they need expensive equipments, of tens of thousands euros. For instance, the Michelson heterodyne architecture can reach typically sub-nanometric resolution and repeatability over millimeters range for nanorobotics systems [1-5]. The Fabry-Pérot architecture, using multi-reflections between a fiber sensor and the target, reports a resolution of $2 \mathrm{pm}$ on a $25 \mathrm{~nm}$ range with a working distance up to $40 \mu \mathrm{m}$ and a bandwidth of $1 \mathrm{kHz}$ [6]. A speckle interferometer can also be implemented to achieve a nanometric resolution over a $100 \mu \mathrm{m}$ range for rough surfaces [7]. White-light (or low-coherence) interferometry is widely used in profilometers where it can achieve a $0.1 \mathrm{~nm}$ resolution; it has been adapted for position 
measurement using fiber optics with a $6 \mathrm{~nm}$ resolution over a $100 \mu \mathrm{m}$ range [8]. Interferometry is widely used too in vibration measurements: for example in the experiment of this paper, we used an old Polytec OFV-303 optical head, which is a Mach-Zehnder with a round trip to the target on one arm and a Bragg cell modulator on the other. It allows frequency comparison to determine the sense and speed of movement thanks to the Doppler effect, and the displacement from the phase with a $85 \mathrm{~nm}$ peak-to-peak limit of resolution and a $0.5 \mathrm{~Hz}$ to $250 \mathrm{kHz}$ frequency range. Nowadays, performances have evolved since then with resolutions below the nanometer and bandwidths beyond the MHz. Digital holography is another optic solution for applications in modal analysis of MEMS (micro-electromechanical systems) [9] and dynamic endoscopy [10] for instance, with a sub-micrometric resolution and a bandwidth of a few $\mathrm{kHz}$. Another noncontact solution is the capacitive gauge; nowadays commercial systems report resolution as good as $0.01 \mathrm{~nm}$ over a $100 \mu \mathrm{m}$ range in static case and $0.02 \mathrm{~nm}$ with a $10 \mathrm{kHz}$ bandwidth in dynamic case [11], after software correction of mechanical errors and non-linearities. New setups are studied to increase the range, such as periodically patterned gauges which display a $25 \mathrm{~nm}$ resolution on fine motion over $100 \mu \mathrm{m}$ and a $260 \mathrm{~nm}$ resolution on coarse motion over $1.6 \mathrm{~cm}$ [12]. Longer range solutions include optical encoders with a nanometric resolution within a signal period and a repeatability better than $0.5 \mu \mathrm{m}$ over tens of centimeters [13-15]. However they remain expensive (thousands of euros). The triangulation technique offers a good compromise too, with a resolution and a repeatability of $10 \mathrm{~nm}$, a range of a few millimeters and a sampling frequency up to $50 \mathrm{kHz}$ (vibration measurement up to $25 \mathrm{kHz}$ ) [16].

Over the past decade, fibers optical sensors (FOS) for distance measurements have been extensively developed due to their resolution of a few nanometers, low encumbrance and low cost. They can be used in many domains such as robotics, biomedical, translation plates for AFM or lithography and in harsh environment, such as cryogenics for supraconductors devices [17]. They have also been adapted to vibration measurement through intensity modulation with a bandwidth of $8.5 \mathrm{kHz}$ and a resolution of $10 \mathrm{~nm}$ peak-to-peak [18-19]. Most of them consist of one emission fiber and one or several reception fibers around, collecting the light reflected by the target. Their displacement range is therefore limited by the reception fibers diameter, typically a few hundred micrometers. It can reach longer ranges by implementing several circles of reception fibers [20] but increasing the number of fibers may increase the environmental noise level; another proposal can reach centimetric displacements by addition of a large grating [2122 ] but at the cost of resolution ( 15 to $27 \mathrm{~nm}$ ) due to the angle between the displacement axis and the sensor axis. Finally, a hybrid setup was made with a sensor based on the same principle of reflection photometry, but where the emission fiber is replaced by a Vertical Cavity Surface Emitting Laser (VCSEL) and the reception fibers are replaced by photodiodes [23], which features a larger intrinsic range but a limited resolution. Some works based on fiber ring resonators have also been developed to convert displacement to frequency sensitivity with a nanometric resolution demonstration over micrometric range [24].

This non-contact optical sensor based on fiber bundle is largely used in precision mechanics (measurements of straightness and angle deviations) because of the non-contact advantage and relatively cheap design. Nevertheless, the limitation of the resolution and especially the drift of the response make them difficult to use in metrology applications because long-time measurements are not possible without calibration process. Moreover for existing prototypes, high resolution is obtained to the detriment of the bandwidth. In this paper, we report the enhancement of the resolution to a sub-nanometric scale which relies on low-noise electronics, a reference channel, the increase of the reception fibers number and their thermal and 
pressure isolation. We demonstrate also a strong reduction of the long-term drift that makes the sensor able to be used for long-time acquisitions. Furthermore, the bandwidth of tens of kilohertz is also competitive and a setup for vibrometric or high-speed measurements at high frequencies, based on the same FOS, is presented.

\section{Sub-nanometric resolution in static case}

The fiber sensor consists of a central emitting fiber and several reception fibers around which collect the reflected cone of light. A scheme is illustrated in Fig. 1a (more detailed schemes can be found in [18-22]). Our source is a red LED (650 nm), commonly preferred to laser diodes in order to avoid interferences of multiple reflections and because of their low cost and ease of use. The signal measured at the output of the reception fibers by a single Si photodiode is doubly linked to the distance $d$ of the target by Equation 1 (reflected intensity profile) and 2 (illuminated surface of a reception fiber) [25]:

$$
\begin{gathered}
I_{r}=I_{0} \cdot \frac{r_{0}^{2}}{\left(r_{0}+2 d \cdot \tan \left(\theta_{0}\right)\right)^{2}} \cdot \exp \left(\frac{-r^{2}}{\left(r_{0}+2 d \cdot \tan \left(\theta_{0}\right)\right)^{2}}\right), \\
S_{r}=\int d S r\left(d, r, \theta_{0}\right),
\end{gathered}
$$

with $r_{0}$ the emission fiber radius, $\theta_{0}$ the emission cone angle, $I_{0}$ the central intensity at the emission fiber output, $r$ the radial variable and $d S r$ the surface element of a reception fiber. The illuminated surface depends also on the geometry of the sensor (gap between fibers, fibers diameters, see [25] for details).

The typical response of the fiber sensor is therefore a quasi-linear ascending curve up to the complete covering of the receiving fiber radius, and then exponentially descending $[18,21$, 25]. The part of main interest is the ascending section which features the highest sensitivity with regard to displacement. This quasi-linear ascending section goes from $d=75 \mu \mathrm{m}$ to $d=275 \mu \mathrm{m}$ in our configuration. In the following of the paper, the measurements have been done around a nominal point of $100 \mu \mathrm{m}$ to minimize residual power fluctuations of the LED source. Considering equations 1 and 2, it appears that the most important parameters to get a high sensitivity are the Numerical Aperture (NA $=\sin \left(\theta_{0}\right)$ ) of the emitting fiber and the number of reception fibers [26]. We tested a sensor with 1 emitting fiber of $1 \mathrm{~mm}$ diameter and 16 receiving fibers of $250 \mu \mathrm{m}$ diameter; it has a sensitivity 6 times higher than a typical FOS $(47 \mathrm{mV} / \mu \mathrm{m}$ instead of $8 \mathrm{mV} / \mu \mathrm{m}$ ) which has an emitting fiber of $500 \mu \mathrm{m}$ diameter and 4 receiving fibers of $250 \mu \mathrm{m}$ diameter. Note that several commercial manufacturers (for example Omron or Keyence) deliver a large catalogue of bundles with different characteristics of dimensions and numbers of fibers. Specific home-made bundle could be developed but we choose here a cheap (less than one hundred dollars) commercial one from Keyence (FU-23X) to maximize the number of fibers and the collecting surfaces.

For this device, the resolution is defined as the ratio of sensitivity over noise, so that other teams work with high amplification gains behind the photodetector to get sensitivity up to $60 \mathrm{mV} / \mu \mathrm{m}$ [21] and lock-in detection to enhance the Signal to Noise Ratio (SNR) [18, 21, 22, 26]. We chose to increase the sensitivity through the number of reception fibers, and to use low-noise electronics. Moreover, increasing the number of fibers demands a better isolation and mechanical stability to avoid mode coupling and bending losses. It is well-known that fibers are very sensitive to temperature [27]: it modifies the refractive index, which changes the NA, and the fibers lengths and diameters, which changes the sensor geometry and thus the sensibility and the 
range. Temperature has also a non-negligible influence on the electronic parts of the sensor (source, photodiode and amplification circuit) [28]. We selected a commercial source (FS-V31M from Keyence) based on a LED at $650 \mathrm{~nm}$ and internal regulation to minimize power noise. For the detection circuit, we used an OPA606 operational amplifier and low temperature coefficient resistors (25 ppm) in a transimpedance circuit. To limit the dark current, the photodiode is not polarized. A capacitor avoids the auto-oscillation of the OPA606. As the noise sources (shot noises and thermal noises) are proportional to the square root of the measurement bandwidth, the resolution is enhanced when the integration time increases. In our case, with a 400 ms integration time, it lowers down to $3.6 \mathrm{~nm}$.

We added a reference circuit of the light source to enhance the SNR. The aim of the reference path is to normalize the amplitude to avoid power fluctuations of the source. A $1 \mathrm{~cm}^{2}$ Si photodiode and its transimpedance circuit are positioned behind a beamsplitter plate at normal incidence (Fig. 1b). It implies a loss of sensitivity on the reflected signal, but when we calculate the ratio of both reflected (target) and transmitted (reference) intensities, it finally increases the SNR by a factor 2 compared to the setup with a silver mirror, with a resulting resolution of $1.9 \mathrm{~nm}$ rms (Table 1). Finally, we placed the setup in a box made of barson, a special isolating material (barson is mainly rubber and lead) to isolate the fiber sensor from pressure and temperature variations, which make us gain an additional factor 3 over noise and a factor 10 over drift (Table 1). We reach thus a resolution of $0.6 \mathrm{~nm}$ over a $200 \mu \mathrm{m}$ range with a $400 \mathrm{~ms}$ integration time (Fig. 2) and the drift is dramatically reduced to $41 \mathrm{~nm}$ over a full hour (Fig. 3), which is probably due to the residual thermal drift of the tabletop and other mechanical parts. Note that the direction of the drifts depends mainly on the temperature variations and can be then either positive or negative if the distance between fibers and mobile mirror is going up or down, depending on the time of the day when the measurements are launched. This setup could be further compacted by placing only the fiber wound in a small barson box. Moreover, the beamsplitter, the reference photodiode and its transimpedance circuit are compacted $\left(3 \times 3 \times 4 \mathrm{~cm}^{3}\right)$ to be stuck on a target.

\section{High bandwidth for dynamic case}

Former works on vibrometry using fiber sensors were based on amplitude modulation of the source and on synchronous demodulation [18]. The system bandwidth was therefore limited by the high-pass and low-pass filters and was demonstrated up to $8.5 \mathrm{kHz}$ with an accuracy of $2 \mathrm{~nm}$ rms (10 nm peak-to-peak) limited by the SNR of the fiber sensor and of the electronic circuit. Another system using 2 circles of reception fibers has been tested up to $1440 \mathrm{~Hz}$; its originality is to measure the ratio of both reception fibers sets to avoid loss of sensitivity with the increase of the working distance (it exploits the descending part of the FOS characteristic and thus has a limited resolution) [29]. We adapted our setup by increasing the bandwidth and consequently by reducing the gain, using a lower resistor-capacitor couple through the transimpedance cut-off frequency equation [30]:

$$
f_{c}=\sqrt{\frac{G B}{2 \pi \cdot R \cdot\left(C+C_{P D}\right)}},
$$

where $C_{P D}$ is the photodiode capacity, $G B$ is the gain bandwidth product of the operational amplifier, and $\mathrm{R}$ and $\mathrm{C}$ are the resistor-capacitor elements of the transimpedance circuit.

We are able to measure directly the intensity variation without any modulation technique but we need an anti-aliasing filter before our acquisition card to eliminate the high-frequency 
components due to the commercial source (beyond $125 \mathrm{kHz}$ ). The anti-aliasing filter is a MAX274 $8^{\text {th }}$ order Butterworth low-pass filter with a cut-off frequency of $50.2 \mathrm{kHz}$ and a peaking of $1.15 \mathrm{~dB}$ at $40 \mathrm{kHz}$; it amplifies the signal by a factor $3.48(10.8 \mathrm{~dB})$ within its flat bandwidth part. We calibrated the frequency response of all the detection system by lighting directly the photodiode with an intensity-modulated LED. The Bode diagram of the circuit gives a cut-off frequency of $28.2 \mathrm{kHz}$. Therefore the global sensor chain (transimpedance circuit and anti-aliasing filter) shows a cut-off frequency of $38 \mathrm{kHz}$. The signal is then sampled at a frequency rate of $250 \mathrm{kHz}$. We use an additional software filter, a Butterworth bandpass under LabView, mainly to eliminate the environmental and DC components (we do not use the reference channel neither the barson box anymore), and to further isolate the frequencies of interest if needed.

The vibration is produced by an aluminized piezoelectric translator (APZT, reference AE0505D08F from Thorlabs) that we calibrated with the commercial interferometric vibrometer from Polytec described earlier (measurement resolution of $85 \mathrm{~nm}$ ). The PZT displays a cut-off frequency of $2.2 \mathrm{kHz}$ and its amplitude can be measured up to $10 \mathrm{kHz}$. We then tested our modified setup on this APZT (Fig. 4) up to $10 \mathrm{kHz}$ and found that it reproduces well the PZT response measured by the interferometric vibrometer (Fig. 5), with a conversion coefficient of $15.6 \mathrm{mV} / \mu \mathrm{m}$. The limit of detection is better than the one of the interferometric vibrometer and is experimentally evaluated at $28 \mathrm{~nm}$ peak-to-peak, according to the PZT voltage-to- $\mu \mathrm{m}$ conversion rate. According to the noise level, the theoretical resolution should be $10 \mathrm{~nm}$ peak-to-peak, but could not be verified experimentally because of the inaccuracy of our vibrometer. This setup is interesting for many applications in term of resolution, bandwidth and simplicity.

At higher frequencies, we used a vibrating membrane built by the Laboratoire de Génie Electrique de Paris (LGEP) and with a resonance at $27.8 \mathrm{kHz}$ with an amplitude of $3 \mu \mathrm{m}$ peakto-peak. It consists of a long plate of aluminium excited at one end by a high-frequency piezoactuator which is aimed to be implemented in a micro-pump [31]. On this surface, our setup features a $9.2 \mathrm{mV} / \mu \mathrm{m}$ sensitivity and a theoretical resolution of $17 \mathrm{~nm}$ peak-to-peak. Measurements are possible up to $35 \mathrm{kHz}$ with a detection limit of $66 \mathrm{~nm}$ peak-to-peak. The part $a$ of the Fig. 6 shows the acquired signal at $27.8 \mathrm{kHz}$ by our FOS. The part $b$ of the Fig. 6 is the Fast Fourier Transform (FFT) of the signal, demonstrating the good SNR. The resonance frequency was determined with a resolution of $1 \mathrm{~Hz}$ and was found to vary in a $30 \mathrm{~Hz}$ interval centred on $27.8 \mathrm{kHz}$ in agreement with expectations, and a peak-to-peak amplitude of $4 \mu \mathrm{m}$. The small variation of the frequency is due to mechanical variations of the clamping points at the start of excitation; once excited, it stays constant in time. The vibration signal has also been measured with the interferometric vibrometer with results in good agreement.

\section{Conclusion}

In this paper, we reported the improvement of a fiber optics sensor based on one emitting fiber and 16 receiving fibers around. By using low-noise electronics and by implementing a reference behind a semi-reflective plate we gain a factor 2 on the SNR. We additionally placed the fiber optics in a barson box, which enhanced the long term stability by a factor 10 and the SNR by an additional factor 3. The final results are a drift of $40 \mathrm{~nm} \cdot \mathrm{h}^{-1}$ and a resolution of $0.6 \mathrm{~nm}(1 \sigma)$ for $400 \mathrm{~ms}$ integration time. Then the setup has been adapted to high dynamic measurements by correcting the bandwidth of the electronics circuit which reaches $38 \mathrm{kHz}$ with a $15.6 \mathrm{mV} / \mu \mathrm{m}$ sensitivity on an aluminized piezoelectric actuator, without any modulation 
technique. The limit of detection is evaluated at $28 \mathrm{~nm}$ peak-to-peak. Measurements have been made on another unpolished aluminum plate actuated by piezoactuators from LGEP up to $35 \mathrm{kHz}$ with $66 \mathrm{~nm}$ peak-to-peak limit of detection; the resonance frequency at $27.8 \mathrm{kHz}$ was clearly seen. The compactness and the low cost of these fiber sensors, along with their good resolution in amplitude and frequency could be used in applications such as modal analysis and multi-point surface deformation monitoring.

\section{Acknowledgements}

The authors thank Y. Bernard and C. Hernandez from Laboratoire de Génie Electrique de Paris for lending them their high-frequency piezoactuator prototypes. This work is supported by the National Research Agency and the PRES (Scientific Research and Education Pole) UniverSud Paris.

\section{References}

[1] A. Sinno, P. Ruaux, L. Chassagne, S. Topçu, Y. Alayli, G. Lérondel, S. Blaize, A. Bruyant, P. Royer, Enlarged atomic force microscopy scanning scope : novel sample-holder device with millimeter range, Rev. Sci. Instr. 78 (2007) 095107-7.

[2] L. Chassagne, S. Topçu, Y. Alayli, P. Juncar, Highly accurate positioning control method for piezoelectric actuators based on phase-shifting optoelectronics, Meas. Sci. Technol. 16 (2005) 1771-1777.

[3] L. Chassagne, M. Wakim, S. Xu, S. Topçu, P. Ruaux, P. Juncar, Y. Alayli, A 2D nanopositioning system with sub-nanometric repeatability over the millimeter displacement range, Meas. Sci. Technol. 18 (2007) 3267-3272.

[4] S. Topçu, L. Chassagne, Y. Alayli, P. Juncar, Improving the accuracy of homodyne Michelson interferometers using polarization state measurement techniques, Opt. Comm. 247 (2005) 133-139.

[5] I. Misumi, S. Gonda, T. Kurosawa, K. Takamasu, Uncertainty in pitch measurements of one-dimensional grating standards using a nanometrological atomic force microscope, Meas. Sci. Technol. 14 (2003) 463-471.

[6] D. T. Smith, J. R. Pratt, L. P. Howard, A fiber-optic interferometer with subpicosecond resolution for dc and low-frequency displacement measurement, Rev. Sci. Instr. 80 (2009) 035105-8.

[7] M. Virdee, Sub-nanometric precision displacement sensing using low mass and rough surfaces as movable targets, Opt. Las. Technol. 36 (2004) 107-115.

[8] L. M. Manojlovic, A simple white-light fiber-optic interferometric sensing system for absolute position measurement, Opt. Las. Eng. 48 (2010) 486-490.

[9] N. Demoli, H. Halaq, K. Sariri, M. Torzynski, D. Vukicevic, Undersampled digital holography, Opt. Express 17 (18) (2009) 15842-15852.

[10] G. Pedrini, W. Osten, Time resolved digital holographic interferometry for investigations of dynamical events in mechanical components and biological tissues, Strain 43 (2007) 240-249. [11] http://www.physikinstrumente.com/en/products/capacitive_sensor/index.php, D-510 and D-100 products (2010).

[12] S.-C. Lee, R. D. Peters, Nanoposition sensors with superior linear response to position and unlimited travel ranges, Rev. Sci. Instr. 80 (2009) 045109-6. 
[13] LIP372-382 models, Heidenhain catalog (2007).

[14] L. M. Sanchez-Brea, T. Morlanes, Metrological errors in optical encoders, Meas. Sci. Technol. 19 (2008) 115104-115111.

[15] A. Yacoot, N. Cross, Measurement of picometre non-linearity in an optical grating encoder using X-ray interferometry, Meas. Sci. Technol. 14 (2003) 148-152.

[16] http://www.keyence.fr/products/vision/laser/lkg/lkg.php, LK-G10 and LK-G15 products.

[17] X. Hu, Q. Wang, C. Cui, Y. Lei, Output properties of fiber optic sensor for microdisplacement measurement at 77K and 4.2K, Cryogenics 49 (2009) 302-304.

[18] R. Dib, Y. Alayli, P. Wagstaff, A broadband amplitude-modulated fibre optic vibrometer with nanometric accuracy, Measurement 35 (2004) 211-219.

[19] Y. Alayli, S. Topçu, D. Wang, R. Dib, L. Chassagne, Applications of a high accuracy optical fiber displacement sensor to vibrometry and profilometry, Sens. Actua. A 116 (2004) 8590 .

[20] M. Noshad, H. Hedayati, A. Rostami, A proposal for high-precision fiber optic displacement sensor, Proc. Asia-Pacific Microwave Conf. (2006) .

[21] C. Prelle, F. Lamarque, P. Revel, Reflective optical sensor for long-range and highresolution displacements, Sensors and Actuators A 127 (2006) 139-146.

[22] A. Khiat, F. Lamarque, C. Prelle, P. Pouille, M. Leester-Schädel, S. Büttgenbach, Twodimension fiber optic sensor for high-resolution and long-range linear measurements, Sensors and Actuators A 158 (2010) 43-50.

[23] I. Ishikawa, R. Sawada, E. Higurashi, S. Sanada, D. Chino, Integrated microdisplacement sensor that measures tilting angle and linear movement of an external mirror, Sensors and Actuators A 138 (2007) 269-275.

[24] F. Vollmer, P. Fischer, Frequency- domain displacement sensing with a fiber ringresonator containing a variable gap, Sensors and Actuators A 134 (2007) 410-413.

[25] D. Wang, Conception et réalisation d'un minicapteur de déplacement à fibres optiques de résolution nanométrique, Ph.D. thesis, University of Technology of Compiègne (1999).

[26] M. Yasin, S.W. Harun, H.A. Abdul-Rashid, Kusminarto, Karyono, and H. Ahmad, The performance of a fiber optic displacement sensor for different types of probes and targets, Laser Phys. Lett. 5 (2008) 55-58.

[27] L. Perret, P. Pfeiffer, B. Serio, P. Twardowski, Thermal characterization of optical fibers using wavelength-sweeping interferometry, Appl. Opt. 49 (2010) 3601-3606.

[28] Y. Alayli, S. Topçu, L. Chassagne, J. Viennet, Compensation of the thermal influence on a high accuracy optical fibre displacement sensor, Sens. Actua. A 120 (2005) 343-348.

[29] X. Li, K. Nakamura, S. Ueha, Reflectivity and illuminating power compensation for optical fibre vibrometer, Meas. Sci. Technol. 15 (2004) 1773-1778.

[30] Hamamatsu documentation of Silicium photodiode on www.hamamatsu.com.

[31] C. Hernandez, Y. Bernard, A. Razek, A global assessment of piezoelectric actuated micropumps, Eur. Phys. J. Appl. Phys. 51 (2010) 20101-8. 


\section{Vitae:}

Luc Perret received the Engineer degree in instrumentation from ENSICAEN, France, in 2003, and the Ph.D. degree in photonics from the University of Strasbourg, France, in 2007. After a post-doctoral position at the Laboratoire des Systèmes Photoniques, Strasbourg, on absolute distance interferometry and tunable lasers, he is now a post-doctoral fellow at the LISV where he works on fiber optics sensors for distance and vibration measurements.

Luc Chassagne is graduated from Engineer degree of Supelec (France) in 1994 and received his Ph.D. in optoelectronics from the University of Paris XI, Orsay, France in 2000 for his work in the field of atomic frequency standard metrology. He is now Professor at the LISV and the topics of interest in its research are nanometrology, precision displacements, sensors and AFM instrumentation.

Suat Topçu received his $\mathrm{PhD}$ from Compiègne University of Technology (Compiègne, France) in 2001. Since 2002, he works as an assistant professor at the University of Versailles in LISV laboratory. His fields of research are interferometry, dimensional metrology, ellipsometry and recently laser cooling and trapping process. He is professor at LISV since 2010.

Pascal Ruaux received the Ph.D. degrees in Robotic from the University of Pierre \& Marie Curie-Paris 6, Paris, France in 1998 to LRP (Laboratoire de Robotique de Paris). He became Associate Professor in 2001 with the LISV. He worked on technical assistant to disability. Since 2006, the centers of interest are control micro- and nano-precision on millimeter displacement, AFM large image and nano-manipulation.

Barthélemy Cagneau received the Ph.D. degrees in mechanical engineering from the University of Pierre \& Marie Curie-Paris 6, Paris, France in 2008. After a postdoctoral position in nanorobotics at ISIR (Institut des Systèmes Intelligents et de Robotique, Paris), he became Associate Professor in 2009 with the LISV (Laboratoire d'Ingénierie des Systèmes de Versailles, Versailles). His research interests include force control, adaptive control, and robust bilateral couplings for micro- and nano-robotics.

Yasser Alayli received his $\mathrm{PhD}$ in applied physics from Pierre et Marie Curie University of Paris (Paris, France) in 1978. He is professor at Versailles Saint-Quentin University, France, and director of the LISV. His research interests include precision engineering domain with subnanometric accuracy and nanotechnologies. 
(a)

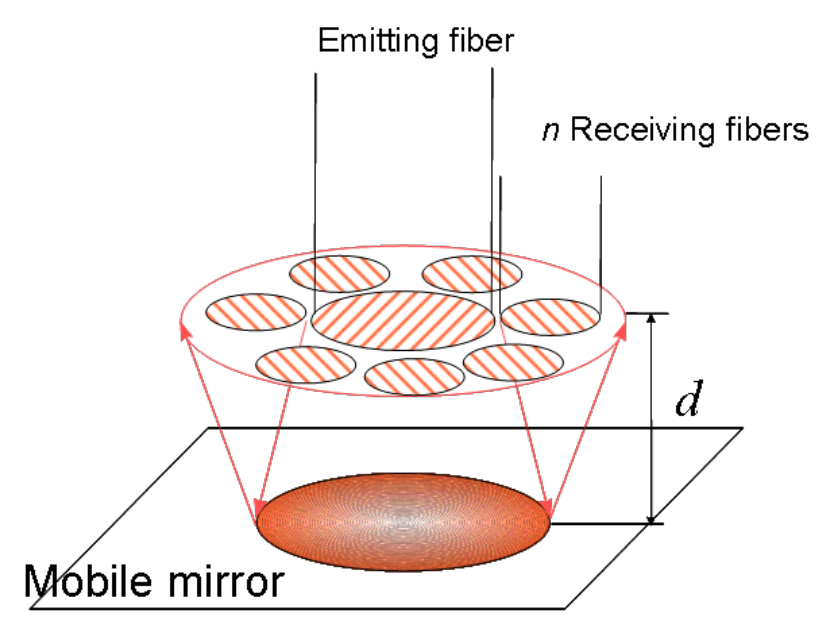

(b)

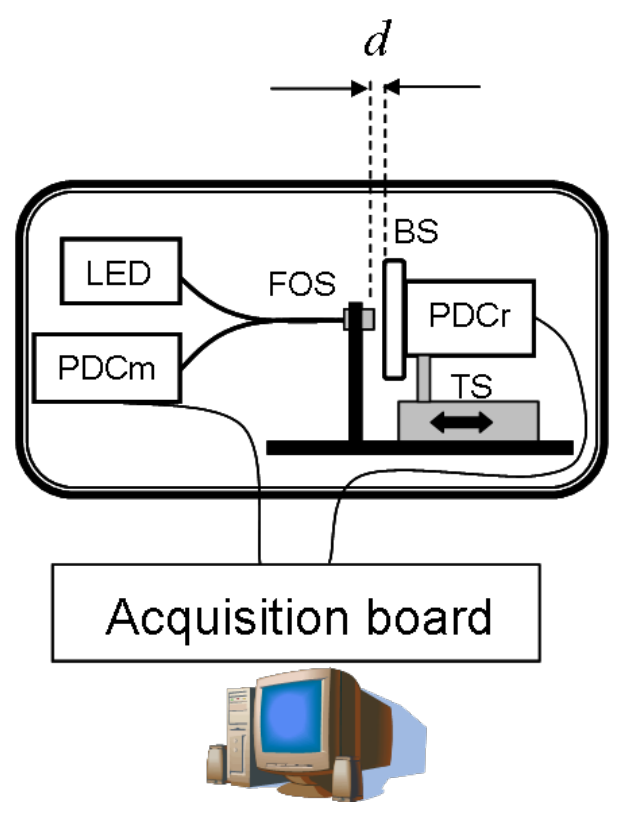

Figure 1 


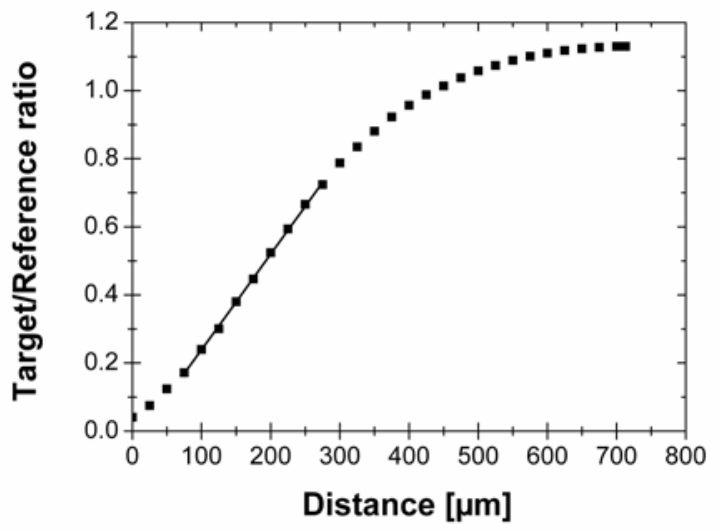

Figure 2 


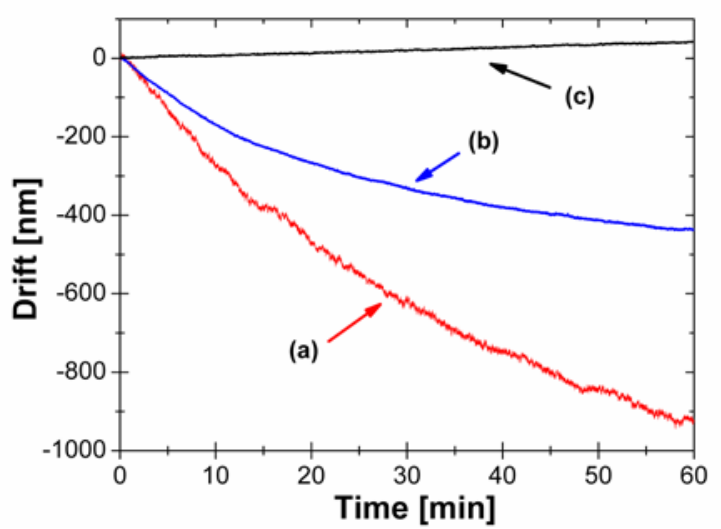

Figure 3 


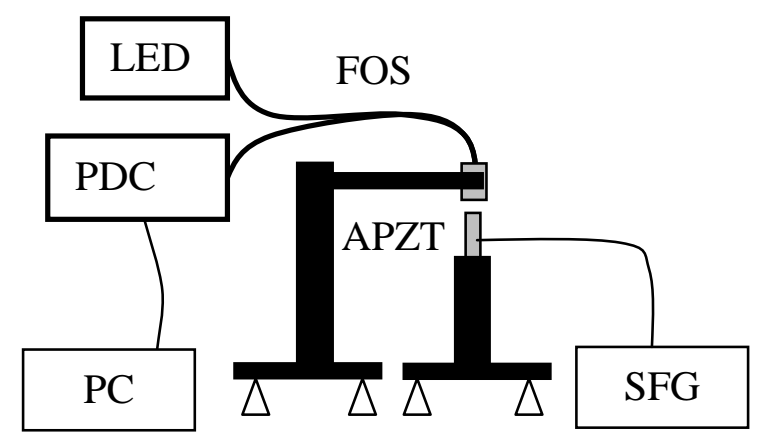

Figure 4 


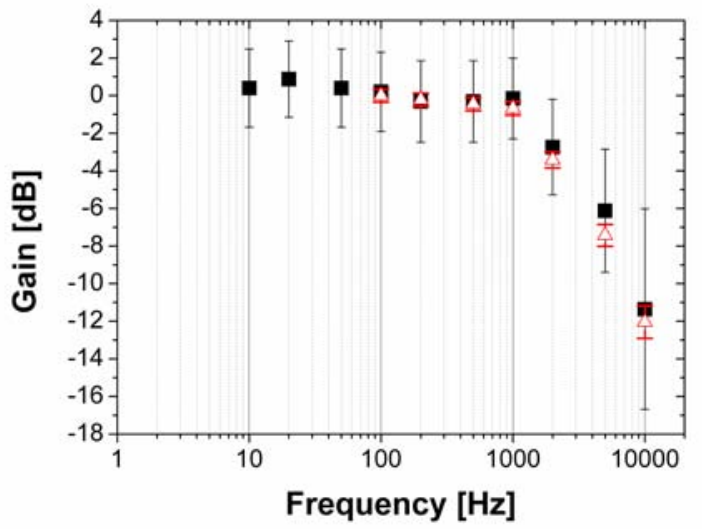

Figure 5 


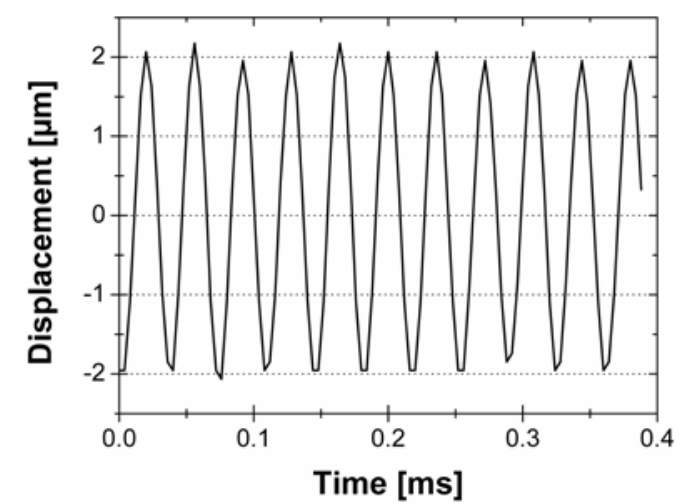

(a)

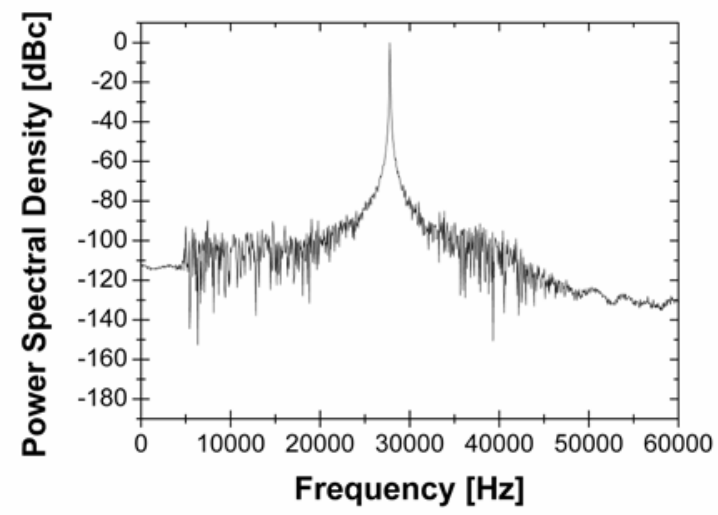

(b)

Figure 6 


\section{Figure caption}

Figure 1: Setup of the sensor measuring the distance $d$. (a) Scheme of the fiber bundle disposition. In our experiments, $\mathrm{n}=16$, diameters of the emitting and receiving fibers are $1 \mathrm{~mm}$ and $250 \mu \mathrm{m}$ respectively. (b) Setup using a reference channel; BS: beam splitter, PDC: photodiodes and their transimpedance circuits, FOS: fiber optics sensor, TS: translation stage (manual or PZT). The whole setup can be placed into an isolating box as drawn.

Figure 2: Ratio characteristic with the reference channel and an integration time of $400 \mathrm{~ms}$; dot: experimental data, line: linear part of interest (linearity of $1.33 \%$ ) with a coefficient of $2.8 \times 10^{-3} \mu \mathrm{m}^{-1}$ over a range of $200 \mu \mathrm{m}$.

Figure 3: Drift of the fiber sensor over 1 hour (integration time for each point is $400 \mathrm{~ms}$ ). (a) on the measurement channel (reflection from the beamsplitter), (b) ratio of the measurement and reference channels, (c) ratio inside an isolating box.

Figure 4: Setup for the vibrometric measurement. FOS: fiber optics sensor, APZT: aluminized piezoelectric translator, SFG: synthesized function generator, PDC: photodiode transimpedance circuit (neither reference nor barson box in this setup, but two different suspended tabletops to avoid vibration propagation).

Figure 5: Bandwidth of the aluminized piezoelectric translator evaluated with a commercial vibrometer (squares) and with the fiber sensor (triangles).

Figure 6: Acquisition at the resonance frequency $(27.791 \mathrm{kHz})$ of the LGEP vibrating plate; (a) the corresponding displacement amplitude is 3.9 (6) $\mu \mathrm{m}$ peak-to-peak, (b) the FFT of the corresponding signal. 


\begin{tabular}{|l|c|c|c|c|}
\hline \multicolumn{1}{|c|}{ Setup } & $\begin{array}{c}\text { Standard } \\
\text { deviation }\end{array}$ & Sensitivity & Resolution [nm] & Drift [nm.h ${ }^{-1}$ ] \\
\hline $\begin{array}{l}\text { Direct reflection from } \\
\text { a silver mirror }\end{array}$ & $0.169 \mathrm{mV}$ & $47.2 \quad \mathrm{mV} \cdot \mu \mathrm{m}^{-1}$ & 3.6 & $>1000$ \\
\hline $\begin{array}{l}\text { Direct reflection from } \\
\text { the beamsplitter plate }\end{array}$ & $0.144 \mathrm{mV}$ & $13.8 \quad \mathrm{mV} \cdot \mathrm{\mu m}^{-1}$ & 10.4 & 707 \\
\hline $\begin{array}{l}\text { Target/Reference } \\
\text { ratio without barson }\end{array}$ & $5.4 \times 10^{-6}$ & $0.0028 \mu \mathrm{m}^{-1}$ & 1.9 & 437 \\
\hline $\begin{array}{l}\text { Target/Reference } \\
\text { ratio with barson }\end{array}$ & $1.8 \times 10^{-6}$ & $0.0028 \mathrm{\mu m}^{-1}$ & 0.6 & 41 \\
\hline
\end{tabular}

Table 1

\section{Table captions list}

Table 1: Standard deviation, sensitivity, resolution and long term drift for different setup of fiber optics position sensors based on 1 emission fiber and 16 reception fibers; integration time $400 \mathrm{~ms}$. 